\title{
Visualizing individual differences in Web navigation: STRATDYN, a tool for analyzing navigation patterns
}

\author{
BETTINA BERENDT and ELKE BRENSTEIN \\ Humboldt University, Berlin, Germany
}

\begin{abstract}
Navigational behavior on the Web can be analyzed with different methods. Log file data are an important source of behavioral traces of navigation. In this paper, we first discuss existing approaches to the classification and visualization of movement sequences that are important for understanding Web navigation. We then present STRATDYN, a tool that provides meaningful quantitative and qualitative measures from server-generatedlog files, as well as easy-to-follow visualizations of navigational paths of individual users. We demonstrate the usefulness of this new approach by reporting the results of two studies (with 44 students in education and vocational training), which show that navigational effectiveness is positively related to the ability to concentrate and selectively focus attention, as measured by the D2 Test of Attention and the FWIT, a German version of the Stroop test. Finally, we discuss implications for further research in this area and for the continuing development of the approach presented.
\end{abstract}

The questions of how people navigate through hyperspace, what decisions they make, and why they make these decisions are important for those concerned with computer-mediated learning, as well as for interface designers and authors of hypermedia information spaces. Although users are nowadays more familiar with hypertext and the quality of hypermedia design continues to improve, users still complain about "being lost in hyperspace" and suffer from cognitive overload (Edwards \& Hardman, 1989; Wright, 1991). The question as to why users may have problems when navigating in complex information spaces can be addressed with a broad spectrum of research methods, such as think-aloud protocols, interviews, or questionnaires (Chen \& Rada, 1996; MacGregor, 1999). However, owing to the inherent shortcomings of self-report data, it is desirable to obtain "objective" behavioral data regarding navigational paths and user actions, which can be provided by server log files. However, obtaining, preparing, analyzing, and interpreting such data are not easy. Getting a full recording of participants' Web interactions is the first hurdle facing researchers (Ellis, Jankowski, Jasper, \& Tharuvai, 1998). The biggest challenge, however, usually lies in analyzing and interpreting log file data. Although methodological proce-

The authors thank Alexandra Biegay, Dörte Felsing, Wenke Haase, Ute Ruprecht, and Kornelia Werner, psychology students at the University of Potsdam, for their contribution in planning and conducting the studies and our reviewers for helpful suggestions on earlier drafts. We appreciate the support obtained from the development teams of the German Education Server and SchoolWeb and the supporting institutions-Humboldt University Berlin, DFN-Verein, DIPF, and FWU (see http://www.eduserver.de/impressum.html). Correspondence concerning this article should be addressed to B. Berendt, Humboldt University Berlin, Institute of Pedagogy and Informatics, Geschwister-Scholl-Str. 7, D-10099 Berlin, Germany (e-mail: berendt@educat.hu-berlin.de). dures have been developed for visualizing and analyzing large-scale log file data for data mining purposes (e.g., Spiliopoulou, 1999), few effective procedures exist for the psychological researcher interested in the navigational behavior of individuals in response to different task demands or conditions (Unz \& Hesse, 1999). In this paper, we present a new approach to the classification and visualization of navigation behavior that provides the researcher with quantitative measures, as well as with an easy-to-follow graphical representation for detailed qualitative analy sis.

In the following, we first discuss existing approaches to the classification and visualization of movement sequences that are important for understanding Web navigation. Second, we discuss requirements for the visualization of individual navigation graphs and then present the approach taken with the tool STRATDYN. Third, we show that individualdifferences in the ability to selectively focus attention when navigating the Web can be analyzed with the help of the qualitative and quantitative data generated by STRATDYN. Finally, implications for further research in this area and the continuing development of the approach presented are discussed.

\section{FORMALIZATIONS OF NAVIGATION BEHAVIOR}

Navigation behavior is often formalized in terms of measures. A measure is a one-dimensional variable computed from the recorded navigation history-in our case, Web server logs. Typical measures include the total number of pages visited to solve a task (e.g., Kim, 2000; Wandke \& Hurtienne, 1998), the total time needed to solve a task, average times spent on single pages (e.g., Hall, Balestra, \& Davis, 2000), or extent to which pages supposed to be accessed together were in fact accessed together (e.g., 
Hall et al., 2000). However, such measures treat navigation histories as essentially linear entities, neglecting the nonlinear structure of hypertext and navigation sequences, where revisits to nodes are common.

The modeling of navigation as a graph preserves this information. A Web site, or another set of hyperlinked pages, itself can be modeled as a graph (or node-and-link structure; cf. Conklin, 1987). Navigation traverses this graph, visiting some nodes but not others, revisiting some nodes, but considering other nodes only once each in long linear sequences. In this traversal, patterns may exist. Patterns can be particular sequences of nodes (e.g., [home page, general-info-page]) or more complex regularities in behavior. For example, one may look for "routes through the data that do not cross any node twice" as opposed to "routes through the data that return to the node where they started." The occurrence of such patterns can be counted, giving rise to indices like "pathiness" or "ringiness," used to denote the number of such routes by Canter, Rivers, and Storrs (1985). So if patterns can be rigorously specified, they define measures for the data.

Here, we model navigation behavior in terms of navigation graphs. A navigation graph is a directed graph consisting of one node per distinct URL visited and one edge (link) per movement from one URL to the next. Each edge is labeled by the number of the step at which this movement is made.

\section{Navigation and Search}

Formalization as a graph is related to modeling in the problem-solving literature (e.g., Newell \& Simon, 1972). There, the search ${ }^{1}$ for the solution of a problem is described in terms of a search tree whose nodes need to be generated by the problem solver. A tree is a more restricted structure than the graph we have introduced above: It is a directed acyclic graph in which each node has exactly one incoming edge, with the exception of the root node, which has no incoming edges.

Search proceeds by traversing this search tree. Either a visited node is a goal node, or the next node to be visited needs to be determined. This choice can be made in "systematic" ways that are independent of any domainspecific information. Such blind search techniques rely solely on the positions of the nodes in the search tree. Blind search techniques are designed to search a tree exhaustively. The problem is that, even for a modestly sized tree, finding a solution may take very long. The choice of the next node to be visited can also be made dependent on domain-specific information, such as the content of the nodes and the task at hand. Such heuristic search techniques often reach the goal after a small number of steps, but they are not guaranteed to find a goal node, even if one exists.

The best-known blind search techniques are depth first and breadth first. In depth-first search, each possible path in the search tree is followed to the end. If no solution is found, backtracking to a higher level occurs, and another path is followed to the end. In breadth-first search, each level is searched completely before nodes at the next level are examined. So blind search can be described by simple algorithms. If an evaluation function can be formally specified that ranks the nodes that are candidates for the next visit, heuristic search too can be described by an algorithm. For example, in the popular hillclimbing heuristic search technique, a set of candidates is first evaluated in terms of each node's likely contribution to the goal, before the node with the highest value is chosen. However, as we will discuss in the next section, both the presence and the nature of an evaluation function in human search behavior may be difficult to identify.

One of the main goals of a search is to reach a goal quickly - that is, to minimize the effort caused by the generation and evaluation of nodes. Quick searches usually involve a small number of nodes generated. However, in a rich information space, another goal may be to acquire extensive knowledge about this space. Although the Web server logs only give us data about the sequence of pages seen and the search terms entered-that is, navigation paths - we can use these data, with appropriate caution, to make inferences about the knowledge structures constructed. The extent of navigation preferably in combination with response latencies (how many pages were seen, how much time the user spent viewing them) can serve as a rough indicator for how much of the information was "covered" (Schellhas \& Brenstein, 1998).

\section{Web Searches}

How can general search theory be applied to the description and analysis of searches on the Web? First, the notion of a Web search should be explicated. In general, any traversal of a set of Web pages, in search of specific information, qualifies as a Web search. ${ }^{2}$ A more specific meaning is the attempt to find Web pages relating to one or more keywords through the use of a search engine like Google (http://www.google.com) or of a Web index like Yahoo (http://www.yahoo.com). Search engines prompt the user for one or more typed-in keywords and return a set of pages deemed relevant because of syntactic analysis (occurrence of the keyword[s] in the full text or in the HTML meta tags, evaluation of links pointing to this page, as in Google's PageRank method, etc.). Web indices typically offer the user a hierarchical menu of categories (like "education," "sports," "real estate," etc.), leading to lists of hyperlinks deemed relevant to the category by human experts. Web portal is another term used for Web indices, usually with a focus on specific content. The differences between search engines, Web indices, and Web portals are fading: Most of today's search engines also offer category menus, and most indices and portals offer at least some keyword specification and full-text search. Users employ both keyword specification and looking through linked lists of pages. ${ }^{3}$

All of these entry points into the Web are largely meta servers, linking to content pages distributed across servers all over the world. A central factor of their usefulness is that they organize the highly heterogeneous material that 
they point to, and often describe the linked resources in sufficient detail for a survey view of the topic of interest. For example, the German Education Server, used in the study described below, offers a detailed description of each of over 9,000 on-line teaching/learning resources from its database. Thus, it is often easier to find relevant details about a resource on the German Education Server description page than on that resource's own pages. This shows that Web searches may, but do not have to, extend across several servers. A Web search may remain completely within one server, depending on the size and comprehensiveness of that server and the nature of the information sought.

Web searches within one server or across several servers can be represented and analyzed in the same manner: In either case, they consist of a sequence of URL requests that may be modeled in the ways described below. This is because, in each case, search operates on a Webset. A Webset is a set of pages potentially relevant for the information sought. The Webset may be a list of pages returned by a search engine, plus the search engine's page containing that list, which may be used for refining the search terms, and so on. The Webset may also be the home page or a deeper level page of an index or of a portal, plus all the pages reachable from it. All Websets are subgraphs of the whole Web and therefore share the same basic structure. The technical caveat is that Web searches within one server can be reconstructed from that server's log files, whereas Web searches across several servers require either the integration of several Web servers' log files (difficult if different organizations host these servers) or data recording by the client or by a proxy server (see Srivastava, Cooley, Deshpande, \& Tan, 2000, for a survey of data collection methods).

What happens during a Web search? The general description of the procedure of searching for a solution to a problem can be transferred to analyses of Web navigation. The initial node is the Web page that a user starts from. This is typically the home page of the server chosen as entry point, whether this is a search engine, a Web index, or a portal. The goal node is a node where the user may stop because he or she feels that enough information has been gathered to answer the initial question. All moves to another Web page are possible moves. They are usually initiated by (1) the activation of a hyperlink or a form submit button, (2) the browser back button, or (3) the browser history. Less frequent is the use of bookmarks or the typing of a URL (Tauscher \& Greenberg, 1997).

In their pure forms, blind search techniques are-for any but the smallest Websets-employed mainly by programs, such as Web robots. Most human Web searches are likely to employ a combination of blind search and heuristic search techniques. On the one hand, the likely contribution of an option to the goal is assessed: A hyperlink is clicked on because its anchor text appears to be semantically closest to, or at least related to, the topic of interest. A keyword or set of keywords is typed in that appears relevant to the topic of interest. Formalizing this assessment of likely contributions amounts to specifying an evaluation function. An evaluation function of Web pages could be similar to the page ranking employed by search engines, where rank increases if the original search terms occur in the Web page's URL, title tag, and so forth. So because human Web searches are directed toward a goal and the descriptions of the next pages reachable from one page are often informative enough to serve as a basis for ranking, some heuristic search will usually be employed.

However, sometimes it is difficult to evaluate and rank the different contributions of possible next nodes. In these cases, a user can only choose the next node at random or resort to a systematic, domain-independent traversal of the available options - that is, employ blind search techniques. Also, evaluating the merit of a page for one's goals may take longer and/or require more effort than clicking on the hyperlink, waiting for the page to load, reading it, and, if it is not the right one, clicking the back button. This is known as the base-level/meta-level tradeoff in AI search theory (Ginsberg, 1993, p. 28). The same idea is expressed in the optimal information foraging theory that applies biological modeling of animals' food foraging to the search for information on the Web (Pirolli \& Card, 1995). This tradeoff leads to (at least partial) reliance on blind search techniques. This behavior may be affected by individual differences in cognitive style. For example, Dufresne and Turcotte (1997) have shown that field-independent users first browse to gain a survey view of a Webset and then return for detailed reading, whereas field-dependent users seem to follow a more sequential path and read fewer pages. First gaining a survey view involves elements of breadth-first search, whereas descending down a branch first involves elements of depth-first search. Here, "involving elements of" means that the first strategy will usually not involve every link from the start page and the second strategy will not descend to the end of one or even all branch(es). In the following, we will refer to such sequences as breadth/depth-first search patterns. (Tauscher \& Greenberg, 1997, distinguish depth-first and hub-and-spoke strategies, which correspond to this distinction.)

A further reason for concentrating on these patterns is the problem of formalization. Different data collection methods (e.g., video recording, think-aloud protocols, and interviews) and analysis methods (e.g., qualitative content analysis) are needed to find out whether a user has evaluated nodes to be visited next, what the evaluation function has been, and whether he or she has really picked the node with the highest value. In other words, it will often be difficult to formalize the reasons for regarding one candidate node (one page linked from the current one) as more likely than another one to lead to the goal. Therefore, a formal approach to the analysis of Web navigation should start by concentrating on the structurally identifiable navigation patterns.

This kind of analysis can be employed regardless of whether the Webset involves pages from one or from several servers. A pattern in a user's navigation history may 
involve nodes from one server, or it may contain nodes from several servers. A user may also repeat the same pattern on different servers-for example, to try to gain a survey view first on each new server.

\section{Search and the Spatial Metaphor}

In a search tree as defined above, the main movement is "forward," toward the goal. In physical (and electronic) space, movements are in various directions, often returning to previously visited locations that serve as useful starting points for exploration. These locations are known as landmarks. Knowledge about a space may extend beyond landmarks to encompass the routes one has already traveled or to include a survey representation of the entire space, which allows one to also infer and take shortcuts. The evolution from landmark via route to survey knowledge is observed both in the cognitive development of children and in the development of adults' knowledge of a new physical space (Siegel \& White, 1975). This taxonomy has also been applied to Web spaces (e.g., Chen, 1999; Dillon, McKnight, \& Richardson, 1993). Landmarks in physical as well as in electronic spaces have three aspects (Sorrows \& Hirtle, 1999): visual, cognitive, and structural. Visual refers to the visual uniqueness of the location, cognitive to the meaning, and structural to the importance derived from role or location in the structure of the space.

The spatial metaphor suggests the use of visualizations, "maps" of the terrain navigated. We will show next that this also allows one to capture individual behavior in more complex ways than can be done with the measures mentioned above.

\section{Patterns in Navigation Behavior}

A pattern is a regularity in behavior, formulated as a valid expression in a knowledge representation scheme (Witten \& Frank, 2000, p. 57). Data are examined with respect to whether they match a pattern template or not. Of particular interest here are sequential patterns. Such patterns can be identified in exploratory analysis (e.g., Olson, Herbsleb, \& Rueter, 1994; cf. Spiliopoulou, 1999, for an example of the analysis of large-scale Web usage data). They can also be identified in confirmatory analysis (e.g., Fu, 2001). Pattern templates are often described in grammatical form-for example, by specifying the identities of the first and the last node in a sequence of requests. Pattern knowledge representation schemes can treat the underlying data as sequences or as graphs. ${ }^{4}$

In the present study, we decided to formulate a number of grammatical descriptions as minimal versions of the patterns we investigated. 5 Specifically, we investigated the following:

Breadth-first search patterns. For each node $A$, we identified sequences $\left[A, B_{1}, A, B_{2}, \ldots, A, B_{k}\right]$, with all visits being first visits and $k$ ranging from 4 to 6 . That is, we determined the number of breadth-first search patterns that investigated at least four (five, six) nodes reachable from a given node.
Depth-first search patterns. For each node $A$, we identified sequences $[A, B, C, D, \ldots]$, with all visits being first visits and the number of nodes ranging from four to six. That is, we determined the number of depth-first search patterns that investigated at least four (five, six) new nodes reachable from a given node in an uninterrupted chain.

Landmarks. A node was classified as a landmark if it had more than five incoming edges and these incoming edges were not all from neighboring nodes - that is, the node was not solely the start of a breadth-first search pattern. Specifically, we required that at least one pair of incoming edges should originate from nodes that were at least five steps apart in the counts of their first visits.

Routes. For each node $A$, sequences were identified that were a depth-first search pattern for two, three, or four steps and then returned by reversing this route.

Survey. This counted the number of moves between nodes that were at least 5,10 , or 15 steps apart in the counts of their first visits.

However, these descriptions are only first approximations to the phenomena we are looking for. The use of visualizations allows for more flexibility in the specification of patterns. The use of visualizations along with quantitative measures is pervasive in exploratory data analysis (cf. Tukey, 1977). Visualization combines two advantages: It allows the representation of large and highdimensional data sets in a compact space, and it allows the analyst looking at that visualization to survey that space and detect complex, higher order patterns that often remain unnoticed in a statistical analysis based on numbers (Card, Mackinlay, \& Shneiderman, 1999). ${ }^{6}$ The attempt to completely formalize expected behavior may even impede exploratory analysis (Olson et al., 1994).

Below, we will demonstrate an approach that combines measures designed to characterize a navigation history as a whole with a more localized pattern analysis relying heavily on visualization. An essential question remains: What should be contained in the visualization, and how should it be visualized?

\section{VISUALIZATION OF NAVIGATION PATHS}

Visualization of paths usually occurs either within graphs, in tree shape, depicting the whole site or as a diagram plotting URLs against time. In the visualization of whole sites, the size of the graph is the central challenge. The most common criteria for a well-drawn graph are minimized edge crossings, uniform edge lengths, straight edges, and symmetry. Depending on the application, nodes should be evenly distributed or distributed in space in such a way that nodes with stronger connections are closer to one another and nodes with weaker connections are farther apart (Chen, 1999). To reach these goals, the number of edges may need to be reduced, clustering algorithms may have to be applied to group semantically linked nodes, nodes and edges may have to be displayed in a deliberately distorted way (e.g., explicit or implicit 
focus-and-context views), and interactive techniques may have to be applied to help the user compose a mental representation integrating several perspectives (e.g., Munzner, 1998; for a recent survey, see Chen, 1999). This basic approach can be applied to the display of navigation graphs. For example, Chen computes the transition probabilities between any pair of nodes and uses these as connection strengths. The resulting data can be displayed analogously to a Web site linkage structure. This can show the perceived proximity or semantic closeness of two nodes well, assuming that they are often visited in sequence. However, a transition probability summarizes all transitions between two nodes, ignoring the history of navigation around this transition. This makes them unsuitable for an in-depth analysis of navigation sequences (see also Niegemann, 2000). Graphs that depict movements in the context of the whole site help to detect, for example, available choices that are never taken, but make it difficult to interpret navigation patterns by content, because the placement of nodes in space is governed by the need to distribute all the site's URLs across this space.

Spiliopoulou (1999) used trees generated from a set of different users' navigation paths that share, for example, the same start and end nodes. This means that every single navigation path is, in effect, displayed as a left-to-right chain of nodes and edges. Although this gives a good idea of the behavior of large groups of users, it relies on textual annotations (each node is annotated by the Web page name and the occurrence number within the navigation paths considered). Thus, important aspects of navigation structure, such as the recurrent visits to nodes, are not easily seen. Also, only the position of the nodes along the $x$-axis is meaningful (steps within the navigation paths). The position along the $y$-axis cannot be interpreted.

Hemmje, Kunkel, and Willett (1994) derive semantic hierarchies of Web pages from the graphic representation of the Web site and display the actual navigation history against this "skeleton" of content-oriented search paths. The hierarchies are trees, and they are visualized as threedimensional cone trees, which provide an implicit focusand-context view. Although this is a good support for users (helping them orient themselves and navigate by supplying a semantics-based "you-are-here map"), it is less helpful for the analysis of navigation paths. Repeated visits to one node do not stand out in the visualization, and a possible structure generated by the user's interaction with the hypertext cannot become visible, because navigation is framed within the structure of the Web site as perceived by the content designer.

Another approach is the plotting of URLs against time (e.g., Hasebrook \& Fezzardi, 1996; Schellhas \& Brenstein, 1998). However, time plots can lead to erroneous interpretations of the shape of the graph, which is often meaningless because URLs are arranged in an arbitrary order along the $y$-axis. In some cases, URLs can be ordered along the $y$-axis in a meaningful way (Berendt, 2000; Niegemann, 2000). However, this is not possible for all types of sites or parts of sites, and patterns involving re- visits of nodes are difficult to see. These techniques are therefore less appropriate for supporting exploratory analysis of navigation in parts of a site.

\section{Requirements for the Visualization of Individual Navigation Graphs and Their Realization in STRATDYN}

The discussion above has shown that existing approaches to the visualization of Web navigation pose problems for person- and task-specific analyses. In the following, we have therefore formulated requirements for visualizing individual navigation graphs, which will be described with reference to the tool STRATDYN. This new approach to the analysis and visualization of $\log$ file data enables researchers to compute a number of quantitative measures of navigation behavior, while at the same time offering a concise visual representation of navigation episodes within a single session.

The visualization is generated in two stages. In the graph definition stage, the ordered list of URL requests is transformed into a navigation graph as defined above. Moreover, several quantitative measures (such as number of nodes and edges, the number of backward moves and back button moves, and branching at home page) are generated. In the graph layout stage, the graph is drawn, with configuration parameters determining how many and whose search episodes are aggregated into one graph, whether branching at the start page or branching at lower level pages is emphasized, and so forth. The resulting navigation graphs can display navigation histories of one or several users. In the analyses reported below, we introduce individualized site maps that show all the navigation moves of one user, emphasizing branching at the start page. Visualization requirements are translated into the use of the visual variables (Bertin, 1983; Mackinlay, 1986) shape, position, and color, each encoding a dimension of the data. This small number of visual variables generates additional structure in the visualization by encouraging perceptual grouping. It also allows patterns to emerge, as will be discussed in the Results section.

Number of nodes and extent of navigation. A first requirement for visualization concerns the number of nodes. The number of nodes in most experimental navigation tasks, such as ours, is small, as compared with even a modestly sized Web site's number of nodes. This means that scalability to very large graphs and algorithmic efficiency are of lesser concern in psychological research. In STRATDYN, the visual variable shape is used to encode whether a graphical element is a node or an edge and what the identity of a node is. Each node is visualized by its name. Each movement between two nodes is shown as an arrow. The resulting image can be treated as a survey view, which enables researchers to readily obtain a global view (targeted vs. disoriented navigation; see Figure 1 for an example). The relative size of different navigation graphs encodes the extent of navigation. At the same time, in an enlarged view, paths can be traced and utilized as a basis for content analysis (see Figure 3 
(a) study 1: participant B

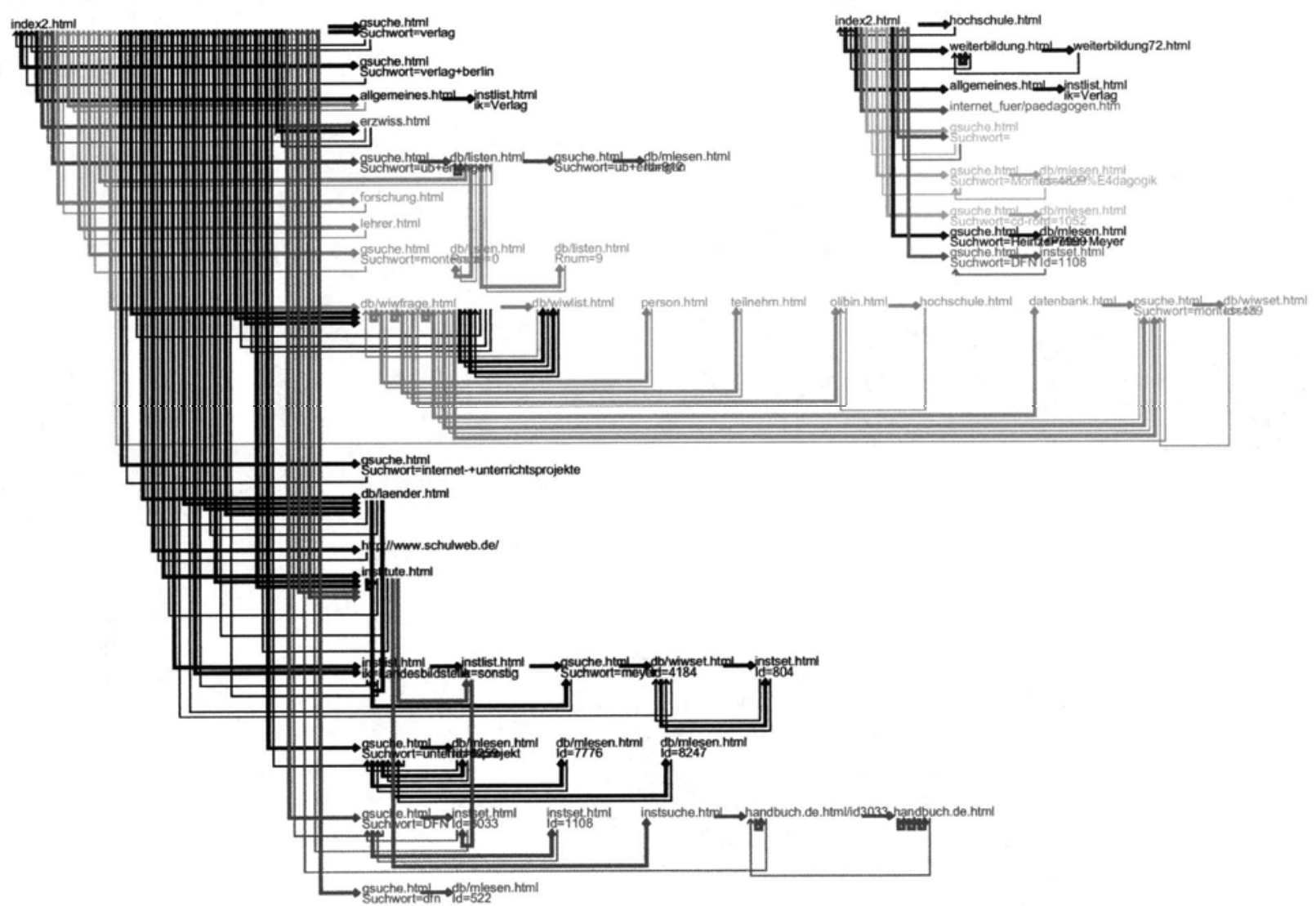

Figure 1. Extent of navigation graphs.

for an example). This tracing of paths, which requires reading the visited pages' names, is not necessary to recognize and understand the extent and structure of the search. For this stage of analysis, individualized site maps can therefore be scaled (as is shown in Figures 1, 4, and 5).

Meaningful dimensions. A second requirement is meaningful dimensions in $x y$ space. By this we mean that the dimensions of the graph drawing - that is, the relative positions of elements in the drawing's $x y$ space-should be interpretable. This is necessary because humans tend to interpret the ordering of magnitudes in a diagram as corresponding to an ordering in reality (e.g., Berendt, Rauh, \& Barkowsky, 1998). In STRATDYN, the $x$ dimension (left-right) encodes temporal order within the processing of one task or the temporal order of visits to one node. Figure 2 shows a fictitious example of seven consecutive moves. The arrows should be read as follows: The first outward move from a node $i$ to a node $j$ is shown by a straight left-to-right arrow if this movement is the first inward move into $j$ (Movements 1 and 5). Otherwise, the movement is shown by the leftmost outgoing edge of the node $i$ (Movements 2 and 6). An outgoing edge has no arrowhead at $i$. The next movement is either another straight left-to-right arrow or the edge immediately to the right of the previous move's incoming end (the end with an arrowhead; Movements 3, 4, and 7). To improve visual clarity, backward moves are also distinguished from forward moves by line width.

The $y$ dimension encodes temporal order as well. First, it (redundantly) codes temporal order of multiple moves to and from a node (see Figure 2, where segments of the edge showing Movement 4 are to the right of and below Movement 3, and similarly for Movement 7 in relation to Movement 6). Second, the $y$ dimension encodes the temporal order of the experimental tasks.

Representation of moves: Exhaustiveness and selectivity. A third requirement is that the graph drawing should combine the advantages of trees with those of full graphs - that is, visual clarity and information richness. In the problem-solving-as-search literature, the traversed graph is a tree. A given node can be the child node of several other nodes, and if it is generated several times in this way, it is duplicated. A graph drawing that duplicates nodes, however, obscures the interconnectedness of problem solving, the reuse of already explored nodes and sub- 


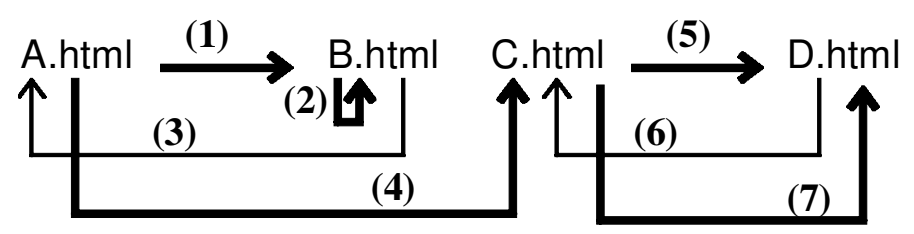

Figure 2. Encoding the order of movements.

graphs. The selective reentry into and exit from previously seen sequences is crucial for understanding the use of a nonlinear text. Nodes should not be duplicated. Another common class of tree representations, spanning trees, does not duplicate nodes but eliminates edges to enhance visual clarity (Munzner, 1998; for pathfinder networks as an alternative way of eliminating edges, see Chen, 1999). A spanning tree of a graph maintains, for each node, exactly one path from a previously chosen root node to that node. This elimination of edges increases visual clarity, but it takes away information. Munzner lets users control the number of displayed edges interactively, thus making that information available.

In STRATDYN, a spanning tree is used as the visualization's basic layout, but at the same time, the full graph is displayed. The visualization offers three independent ways of perceptual grouping. The first grouping shows the nodes in the order of their first visits: The criterion for positioning a node, which is also the criterion for the generation of the spanning tree, is the first visit to a node. This criterion emphasizes the discovery of information. The grouping by task is created by the arrangement in parallel horizontal rows and by similarity of shape: The nodes that are arranged in this basic layout are strings of letters. The second grouping shows all movements, which includes single and recurrent movements that are independent of the order of first visits to the nodes involved. This emphasizes navigation. This grouping is created by similarity of shape: The edges are dominated by horizontal and vertical straight lines (the arrowheads are comparatively small and restricted to the end points of edges, so they do not affect the grouping of edges noticeably).

Representation of nodes and edges: Task structure. The third way of grouping by the visual variable color encodes the experimental task (Task 1, red; Task 2, green; and so on). In halftone printing, the colors appear as different gray values. This is needed as a way of grouping nodes and edges that is independent of the grouping by temporal order. First, each new task involves the opening of a new horizontal branch, but not every new horizontal branch indicates the start of a new task. Second, repeat visits to nodes are particularly interesting if they occur in new tasks, since this indicates possible transfer.

Patterns. A final requirement is the visibility of patterns. The individual moves must be visible, but in addition, we want to be able to identify patterns and to compare their realizations in different navigation graphs. The patterns we are looking for are: breadth-first search patterns, depth-first search patterns, landmarks, route-oriented nav- igation, and survey-oriented navigation. After the identification of patterns, the analyst can proceed to investigate these behavioral sequences qualitatively.

\section{Application of the STRATDYN Approach in Research on Individual Differences in Navigation Behavior}

How this approach works in practice is best illustrated by example. In the following, we therefore show how STRATDYN can be used to visualize different patterns of navigation in a multitask condition and to provide meaningful measures for the quantitative analysis of log file data. To this end, we present selected findings from two experiments in which we addressed the following questions: Do individualdifferences in the ability to focus attention on relevant visual stimuli affect (1) the quality of the interaction as expressed in different navigational patterns, (2) the extent of navigation (i.e., the number of pages visited), and (3) the effectiveness of navigation (number of correct responses in Experiment 1, or the amount of relevant information gathered in Experiment 2).

Following a brief description of the method, we present selected findings to illustrate how the visualizations and measures provided by the STRATDYN approach can aid in answering these questions. A more thorough description of the experiments can be found in (Brenstein \& Berendt, 2001).

\section{Method}

Participants. The samples consisted of 44 German students: 21 university education students (6 male, 15 female; average age, 22) in Experiment 1, and 23 vocational education students (male; average age, 18) in Experiment 2. All the students volunteered to participate for course credit.

Instruments. In both experiments, on-line questionnaires were used to assess students' prior knowledge, general motivation for working with computers, and basic demographic information.

The individual difference variables interference and concentration were assessed with standardized instruments. In Experiment 1, a German version of the Stroop test (Farb-Wort-Interferenztest, FWIT, by Bäumler, 1985) was used to assess participants' ability to ward off interferences.

In Experiment 2, the $d 2$ Test of Attention by Brickenkamp (1994) was used to measure the individual's ability to selectively focus attention. The $\mathrm{d} 2$ is the standard instrument for measuring speed and accuracy in visual stimulus discrimination. In addition to the overall measure of attention (proportion of correct responses), the number of omissions (number of items that participants did not mark) was also assessed.

Tasks. The students were given several tasks in each experiment. The specificity of the tasks was varied within and across experiments. In Experiment 1, the students were given five questions ask- 
ing them to search for factual information on the German Education Server (http://www.eduserver.de). The search tasks were of varying difficulty. In Experiment 2, the students were first asked to search for their school and then for general information in a subject of their choice, to help them prepare for an impending exam. Both searches were conducted on the SchoolWeb server (http://www. schulweb.de). The servers are two large education portals in Germany, offering common interface options of both link following and keyword specification. All information needed to solve the tasks could be found on these servers' pages.

Procedure. The students first completed the assessment of the individual difference variables in individual sessions. They then filled out on-line questionnaires on person variables. Subsequently, the students were asked to complete the respective tasks. The students finally reported their search results and provided quantitative and qualitative feedback regarding their search episodes in another on-line questionnaire. The sessions lasted a total of 60-70 $\mathrm{min}$.

Data cleaning and preparation. The behavioral data were obtained from the Web server access logs of both the SchoolWeb and the German Education Server in the form of a flat file containing one line per page request. The requests were listed in the commonly used Extended Log Format, which contains information on the host (IP address), the requested URL, the date and time of the transaction, and its status or error code (e.g., 200/success, 404/file not found, 301/permanent redirect). Since $\log$ files give rise to a number of uncertainties (e.g., Berendt \& Spiliopoulou, 2000), the data needed to be cleaned and prepared before analysis. First, hits needed to be transformed into page impressions by filtering out, from the Web server log files, all requests for GIF or other image files. Second, the participants had been instructed to turn off their proxies, so their individual computers' current IP numbers appeared in the $\log$ files (IP numbers used in the experiment were known). To segment the sequence of requests from one IP number into sessions of different participants, Web pages controlling the experiment were employed. These asked the participant to "log into" and "log out of" the experiment. Finally, the participants were asked to empty their browser's cache and turn off caching prior to starting the experiment to ensure that the whole sequence of pages seen by the user, including revisits to pages previously requested, was recorded in the log file. Thus, we obtained the full sequence of pages visited on the SchoolWeb or German Education Server for each participant. Intermediate visits to other servers could only be traced via the log file referrer field if the return move was initiated by a hyperlink on the remote page or if a redirect was employed. This was observed only seldom, because the experimental tasks did not require searching for information outside the respective servers (if it did occur, it was not recorded, because participants generally returned via the back button).

Measures. The following measures were used.

1. Extent of navigation. This was measured by the number of nodes and the number of edges. ${ }^{7}$

2. Search strategies. We chose the branching factor of the home page as our indicator of how broadly a user searched-that is, of how much the overall strategy resembled breadth-f irst search. The branching factor equals the number of different new nodes-that is, branches opened up, directly after the home page. Repeat visits were not counted - that is, in a path [home page, X, Y, home page, Y], only $X$ counts toward the branching factor, since this back-andforth movement is interpreted as landmark use rather than as the examination of new ways of answering the question. In contrast, a depth-first navigation strategy explores a path that leads the user away from the start page until the end of that path is reached, or at least until it becomes obvious that this will not lead to the goal. We considered long paths from the home page to be indicative of such a strategy and chose the average depth of exploration as our measure of a depth-first strategy: the number of forward moves divided by the number of branches emanating from the home page. ${ }^{8}$
3. Landmark, route, and survey navigation. We used the number of incoming edges as our measure of landmark use. ${ }^{9}$ To get an overall value for one user, we took the average number of incoming edges (normalized by the total number of nodes). To determine whether a page has visual or cognitive landmark qualities, the page and its contents must be examined. Secondly, we investigated how navigation proceeded. Route-oriented navigation, presumably reflecting reliance on route knowledge, is characterized by many backward moves - in particular, backward moves to the immediate predecessor (mostly using the back button). Survey-oriented navigation, presumably reflecting the availability of survey knowledge, will include more shortcuts - that is, a relatively smaller number of backward moves to the immediate predecessor. It should be noted that survey-oriented navigation in this sense includes the knowledge and skillful use of landmarks.

4. Interface choice. In accordance with Nielsen's (2000) distinction between search- and link-dominant users, we investigated the total numbers and proportions of all actions after the home page of the following: use of the (undifferentiated) search form field on the home page, request for a subsequent search page differentiated by topic, or link following (all other pages; i.e., pages not requiring the typing of a search term).

\section{Results}

Qualitative analysis of navigation paths. The visualizations of individual search paths clearly showed two distinct navigation graphs for students classified as either high or low on measures of interference and attention (e.g., Figure 3 vs. Figure 4).

Figure 3 shows the navigation graph of Participant A in Experiment 1, who had a low value on the interference scale and solved many tasks correctly. Figure 4 shows the navigation graph of Participant B in Experiment 1, who had a high value on the interference scale and solved few tasks correctly. The size of the graphs shows the highly different extents of navigation.

Local patterns can be seen, for example, in the different behaviors in Task 3, a search for a CD-ROM containing information on Montessori pedagogy. The nodes and edges associated with this task are shown in a dark color in the middle of the figure. Participant A pressed the "search" button on the home page without having entered a search term, obtained an error message, and went back to the home page. She then entered a search term, obtained a list of resources, and visited one of these resources. This was done again for another search term. The in-between return to the home page was done in a route-oriented way, retracing the steps taken: Between any two nodes, there is an arrow in the "forward" direction and, if the route was also traced back, also one in the "backward" direction (see sixth row from the top in Figure 3). Search was balanced between breadth-first and depth-first patterns: two branches and a maximal depth of two.

Participant B investigated many different paths in Task 3. This is shown by the broad dark part of the "ribbon" below the home page (top left of the figure). Search involved mainly breadth-first search patterns, returning to the home page immediately on the first five branches. This is shown by the shortness of the dark paths in the horizontal direction (above the part highlighted as "c" in Figure 4). The sixth branch exhibits a breadth-first search 


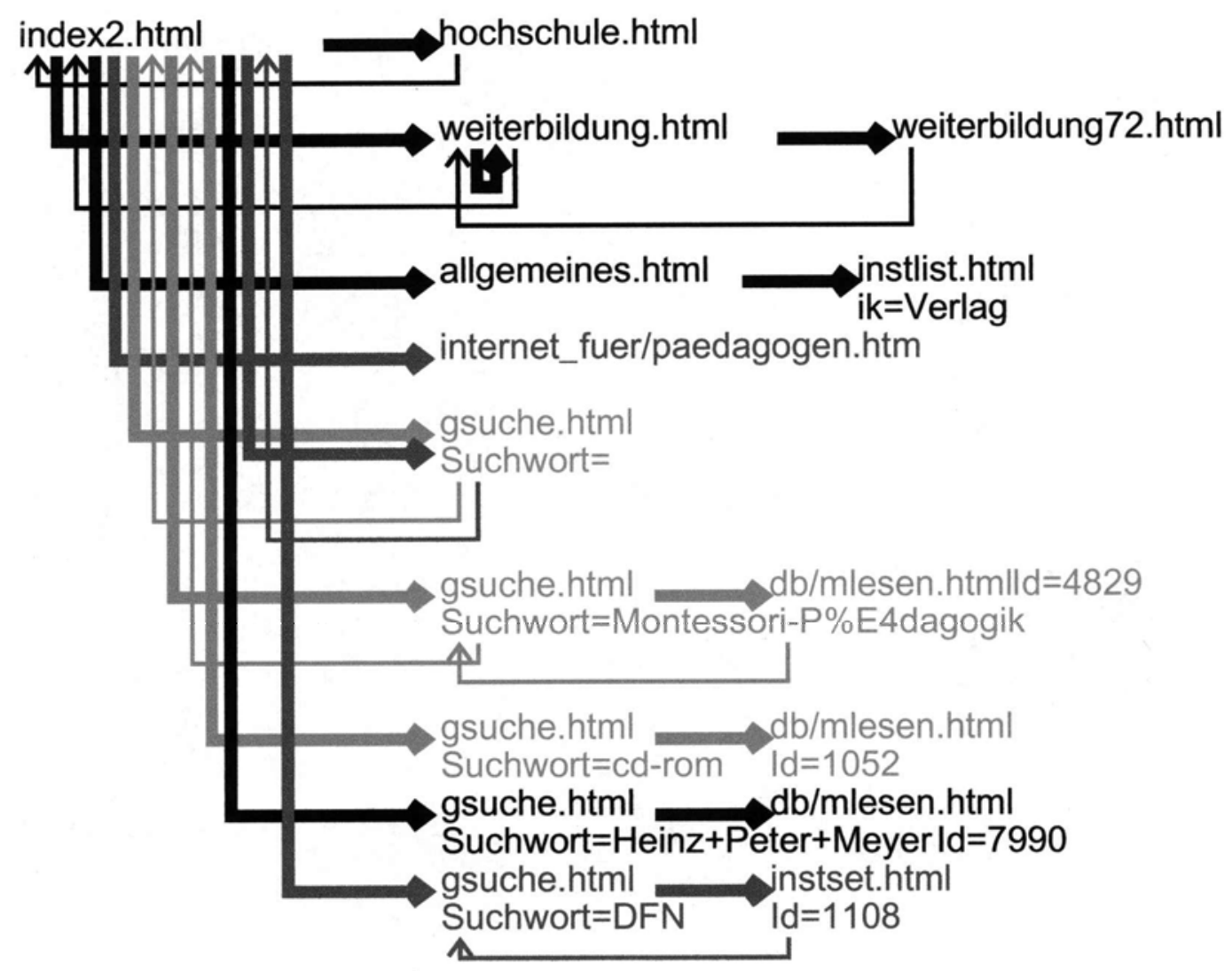

Figure 3. Individualized site map of Participant A in Experiment 1.

pattern at a deeper level, shown by the broad pattern in the middle of Figure 4 (highlighted as "a"). The starting node of this breadth-first search pattern also served as a landmark in B's search history. Landmarks become visible because they have many incoming edges, so they are positioned on top of a "broad ribbon," highlighted as "c." The landmark was reused in Task 4 , a selection of two of the on-line teaching resources provided by a specific author. This is shown by the "ribbon" below the node having different colors/gray values. This is an instance of surveyoriented navigation, which can also lead to vertical movements across task rows (see the lower part of Figure 4). Other nodes were also reused by Participant B. However, most branches ended with a return to the home page, indicating that, on the whole, little survey knowledge was available (Dillon et al., 1993). An instance of a localized depth-first search pattern in Experiment 1 is shown in the part highlighted as "b" in Figure 4. In general, breadthfirst search patterns dominated, giving rise to a high branching factor at the home page.

Figure 5 shows the navigation graph of Participant $\mathrm{C}$ (low on attention) in Experiment 2. Navigation is dominated by depth-first search patterns, shown by the large extent of the branch in the horizontal direction. It should be noted that the SchoolWeb server contains a comparatively large main menu on all pages, encouraging depth- first strategies. The German Education Server, on the other hand, contains much fewer main menu items on its pages. So depth-first patterns were, in general, more distinct in Experiment 2 than in Experiment 1.

The grammatically specified versions of the patterns that could be identified in the data were subjected to a first inspection for frequency of occurrence. The numbers suggested a restriction to breadth-first and depth-first search patterns of at least length 4 , landmarks, routes of at least length 2 , and survey navigation extending across at least a distance of 10. In Experiment 1, this gave rise to a total of 174 patterns, and in Experiment 2, a total of 327 patterns. Table 1 shows that most patterns occurred more frequently in the navigation graphs of participants high on interference/low on concentration. As we have pointed out above, the SchoolWeb server used in Experiment 2 encourages depth-first searching. However, the relative usage of the search patterns varied. In Experiment 1, the participants low on interference used breadth-first and depth-first searching in similar proportions as the participants high on interference $(0.5 / 0.4$ vs. $1.7 / 1.3)$. In Experiment 2 , the participants high on attention relied much more on depth-first searching than did those low on concentration (4.7/0.1 vs. 5/0.3).

As Table 1 shows, there is generally a wide range of occurrences, suggesting that the definition of patterns 


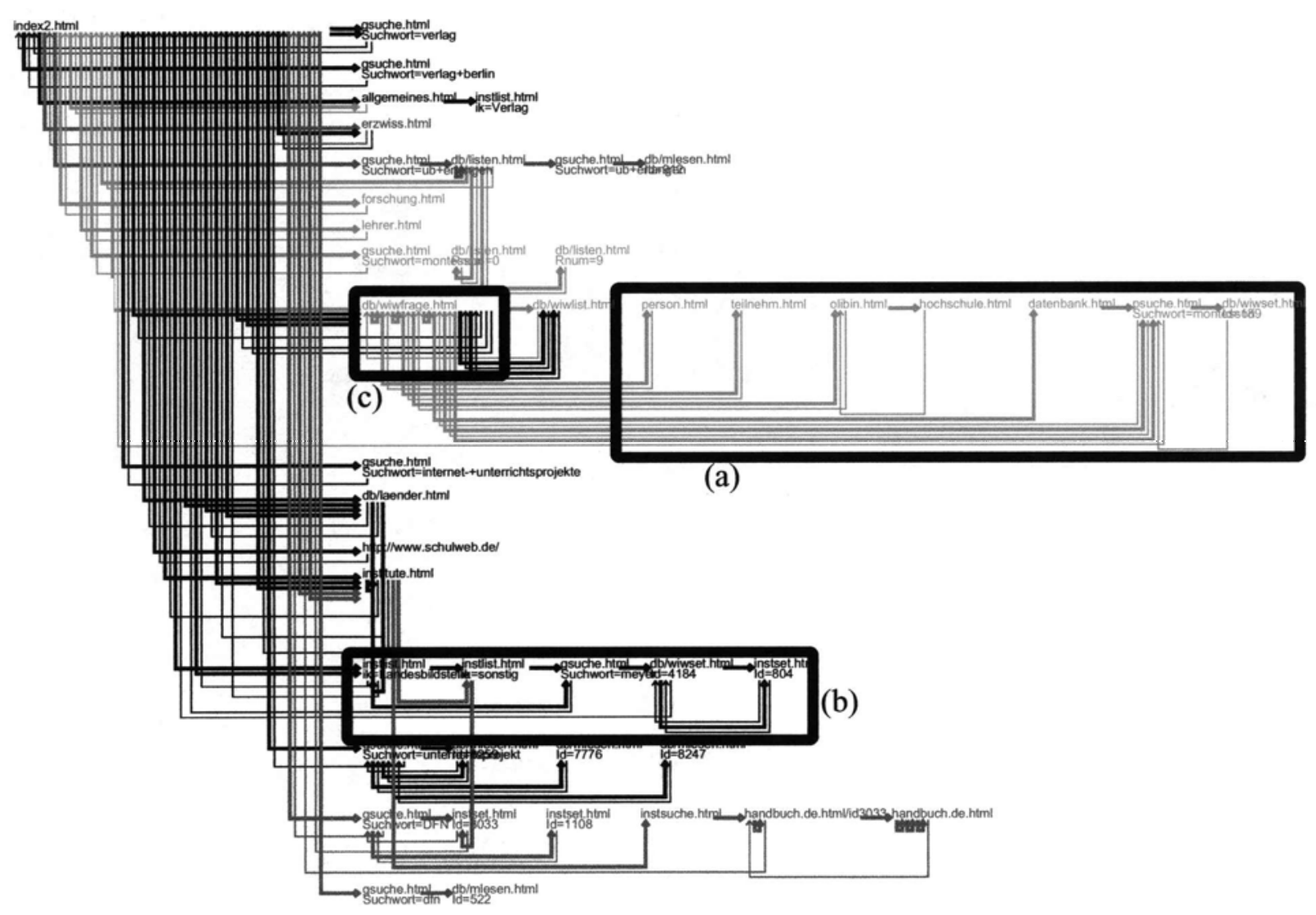

Figure 4. Individualized site map of Participant B in Experiment 1.

needs to be refined and the resulting scheme tested on the basis of more data. In an exemplary way, the above figures illustrate how this could be done. For example, with respect to breadth-first search patterns, we might also want to investigate those that not only visit the immediate next node $B_{i}$, but also one, or two, further nodes before returning to $A$. In addition, we would want to constrain the number of nodes from the $B_{i}$ that may be visited twice. In general, the parameters of path length and node identity should be varied.
Relationships between log file measures and individual difference variables. The observed differences in the qualitative analysis of participants' navigation graphs were statistically confirmed with regard to quantitative measures. Table 2 lists descriptive statistics for the variables discussed in this paper (for detailed results, see Brenstein \& Berendt, 2001).

In Experiment 1, correlational analysis showed that students high on interference had a tendency to request more pages and backtrack significantly more often than did

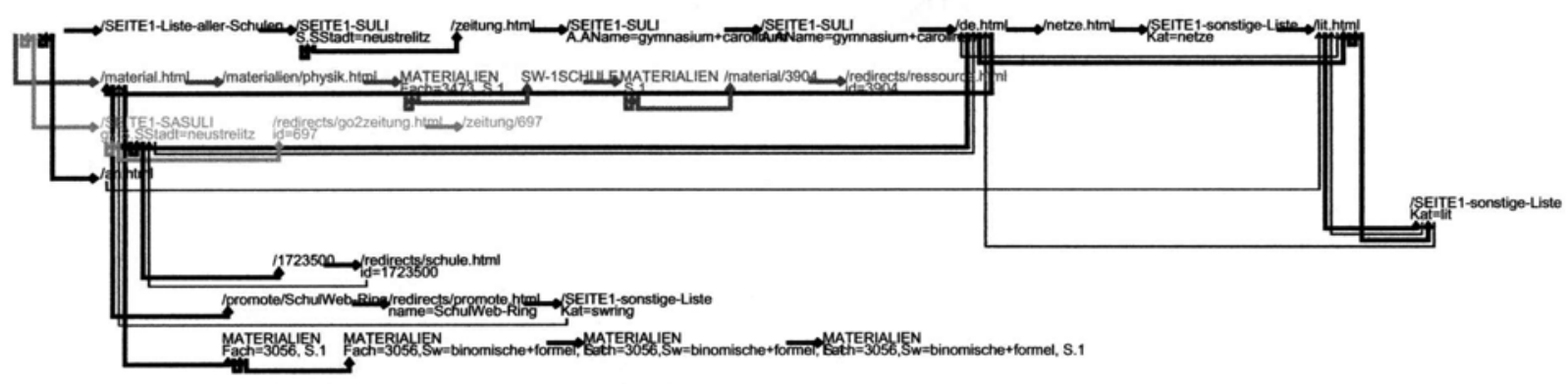

Figure 5. Individualized site map of Participant $\mathrm{C}$ in Experiment 2. 
Table 1

Numbers of Identified Patterns per Person

\begin{tabular}{|c|c|c|c|c|c|c|c|c|}
\hline \multirow[b]{3}{*}{ Pattern } & \multicolumn{4}{|c|}{ Experiment 1} & \multicolumn{4}{|c|}{ Experiment 2} \\
\hline & \multicolumn{2}{|c|}{$\begin{array}{c}\text { Low on } \\
\text { Interference }\end{array}$} & \multicolumn{2}{|c|}{$\begin{array}{c}\text { High on } \\
\text { Interference }\end{array}$} & \multicolumn{2}{|c|}{$\begin{array}{l}\text { High on } \\
\text { Attention }\end{array}$} & \multicolumn{2}{|c|}{$\begin{array}{c}\text { Low on } \\
\text { Attention }\end{array}$} \\
\hline & $M$ & Range & $M$ & Range & $M$ & Range & $M$ & Range \\
\hline Breadth-first & 0.5 & 0.4 & 1.7 & 0.7 & 0.1 & 0.1 & 0.3 & 0.2 \\
\hline Depth-first & 0.4 & 0.2 & 1.3 & 0.3 & 4.7 & 1.9 & 5.0 & 0.1 \\
\hline Landmark & 0.8 & 0.3 & 3.6 & 0.1 & 0.6 & 0.3 & 2.0 & 0.7 \\
\hline Route & 1.3 & 0.4 & 1.9 & 0.4 & 0.3 & 0.1 & 0.6 & 0.2 \\
\hline Survey & 1.1 & 0.8 & 4.4 & 0.1 & 1.0 & 0.8 & 1.5 & 0.5 \\
\hline
\end{tabular}

their more focused colleagues (see Table 3). Interference was not related to depth of exploration in this experiment, which was not surprising, given that students frequently performed keyword specification searches. The negative relationship between the navigational measures and the number of correctly answered questions is also an indication that, in this task condition, extensive exploration was not helpful.

As is shown in Figure 6, students with high interference scores answered significantly fewer questions correctly. This was true for both those who visited many and those who visited few nodes. Students high on interference who visited many nodes were least likely to produce correct results. A two-way analysis of variance (ANOVA), with two groups of students high or low on interference (median split), yielded a significant main effect for the two groups $[F(1,20)=7.567, p<.01]$ when accounting for the overall number of correctly answered questions. As can be seen in Figure 6, no interaction occurred. The results suggest that the ability to ward off interferences does influence outcome measures of factual navigational search tasks.

In Experiment 2, the attention scores were related to branching at home page $(-.56)$ (see Table 4 ), indicating that the participants with higher values on the attention scale tended to visit fewer pages. Also, the number of omissions in the attention test was found to be positively related to the number of nodes visited (.51) and also to the number of wrong search options (.53, not shown in table), suggesting that the participants who were less accurate in visual discrimination tasks on the test visited more pages and were less successful in searching than were those who performed the task more accurately. Detailed qualitative analysis is required to determine whether the visited pages tended to be relevant or irrelevant to the task.

A two-way ANOVA confirmed that the participants with low attention scores branched more at the home page and thus employed breadth-first patterns regardless of the number of nodes visited (see Figure 7). The ANOVA yielded a significant main effect for the two groups $[F(1,16)=4.88, p<.05]$, with no significant interactions. The outcome measures in Experiment 2, which involved an open search task rather than a factual search, are based on a careful content analysis of the written responses. They are discussed in Brenstein and Berendt (2001).

\section{Discussion}

When searching the Web for information, users are confronted with several visual and conceptual discrimination tasks at every point of their search path. In order to stay focused on the task at hand, they have to be able to focus their attention and selectively attend to information relevant to their implicit or explicit query. In this paper, we report first results of two experiments in which we examined whether individual differences in the ability to focus attention on relevant visual stimuli affect (1) the quality of the interaction, as expressed in different navigational patterns, (2) the extent of navigation (i.e., the number of pages visited), and (3) the effectiveness of navigation (number of correct responses in Experiment 1 or the amount of relevant information gathered in Experiment 2).

In both experiments, measures of motivation, prior experience with computers and the Internet, or attitude toward computers did not appear to affect navigational outcomes significantly (this may be partially due to the operationalization of these variables, which is detailed in Brenstein \& Berendt, 2001). However, a positive attitude toward participation in the study tended to increase the depth of exploration. As in most studies, this underscores that more motivated participants engage more in experimental tasks and, in this case, have shown more perseverance in searching for the answers.

The quantitativeresults clearly show that the examined individual difference variables susceptibility toward interference (Bäumler, 1985) and selective attention (Brick-

Table 2

Selected Quantitative Variables Derived from Log File Data

\begin{tabular}{lrrrrr}
\hline \multicolumn{1}{c}{ Variable } & \multicolumn{2}{c}{ Min } & Max & $M$ & $S D$ \\
\hline & \multicolumn{2}{c}{ Experiment 1 } & & & \\
Number of nodes & 21 & 13 & 45 & 27.4 & 11.5 \\
Number of edges & 21 & 20 & 137 & 60.9 & 34.2 \\
Number of backward moves & 21 & 3 & 60 & 22.9 & 15.6 \\
Number of back button moves & 21 & 3 & 41 & 16.2 & 11.5 \\
Branching at home page & 21 & 5 & 22 & 11.3 & 4.9 \\
& Experiment 2 & & & \\
Number of nodes & 23 & 9 & 32 & 21.0 & 6.1 \\
Number of edges & 23 & 12 & 69 & 34.3 & 12.4 \\
Number of backward moves & 23 & 1 & 11 & 5.0 & 3.3 \\
Number of back button moves & 23 & 0 & 22 & 7.1 & 5.1 \\
Branching at home page & 23 & 1 & 7 & 3.0 & 1.4 \\
\hline
\end{tabular}


Table 3

Correlations Between Measures Based on Log File Data and Interference and Number of Correct Responses (Experiment 1)

\begin{tabular}{lcc}
\hline \multicolumn{1}{c}{ Measure } & Interference & $\begin{array}{c}\text { Number of } \\
\text { Correct Responses }\end{array}$ \\
\hline Number of nodes & .38 & $-.51^{*}$ \\
Number of edges & .41 & $-.54^{*}$ \\
Number of backward moves & $.50^{*}$ & $-.57^{* *}$ \\
Number of back button moves & .36 & -.59 \\
Branching at home page & .30 & -.40 \\
Average depth of exploration & .17 & -.32 \\
\hline
\end{tabular}

$* p<.05 . \quad * * p<.001$ (two-tailed).

Table 4

Correlations Between Measures Based on Log File Data and Two Measures of Attention (Experiment 2)

\begin{tabular}{lcc}
\multicolumn{2}{c}{ and Two Measures of Attention (Experiment 2) } \\
\hline \multicolumn{1}{c}{ Measure } & Attention Score & $\begin{array}{c}\text { Number of } \\
\text { Omissions }\end{array}$ \\
\hline Number of nodes & -.11 & $.51^{*}$ \\
Number of edges & .17 & .41 \\
Number of backward moves & .21 & .23 \\
Number of back button moves & .32 & -.25 \\
Branching at home page & $-.56^{*}$ & -.28 \\
\hline
\end{tabular}

$* p<.05$.

enkamp, 1994) affect navigational behavior. In Experiment 1 , we found that students with a higher ability to ward off interferences were more likely to solve questions correctly in a factual search condition. This was true even more so for those who visited fewer pages. In Experiment 2 , the degree of concentration affected the breadth of exploration, in that students who were less accurate in visual discrimination tasks visited more pages. On the other hand, they were less successful in searching. A detailed qualitative analysis is required to determine how relevant the information on the visited pages was to the task at hand.

Chen and Rada (1996) have advocated the use of a more process-oriented approach in hypertext studies, where the sequential structure of user behavior is analyzed on the basis of the paths users have taken when searching or browsing for information. Our approach to the visualization of navigation behavior has enabled us to do just that-namely, to look at the extent of navigation not only from a quantitative point of view (e.g., number of pages visited). The visualizations enabled us to examine examples of successful and less successful navigation histories and to take a look at the specific depth-first versus breadth-first navigation strategies (see also Brenstein, 1996), as well as to distinguish the structural features of individual search paths-landmark, route, and survey approaches-within the context of specific search conditions. Interestingly, the results were stable across different tasks and across the two server environments.

The tasks were representative of the different ways of looking for information in information systems (Shneiderman, 1998). Experiment 1 involved specific and extended fact finding (e.g., finding a CD-ROM title about a specific educational topic). Experiment 2 invited the participants to engage in open-ended browsing and exploration of availability (e.g., finding on-line resources relevant to an impending exam). In an open task like this, the decision as to whether a solution has been found is not always clear-cut but, rather, depends on the depth of understanding a user aims at. Also, the information needed is usually distributed over several Web pages. Although the factfinding tasks were constructed to be meaningful and relevant for students, the open search task in Experiment 2 proved to be more inherently motivating, since it was associated with the real-life incentive of preparing for an impending exam. We found that students approached this task very seriously and often engaged in extensive exploration. Still, the overall influence of the individual's ability to focus attention was similar across these tasks.

In both experiments, we found that the higher the ability to focus attention, the smaller the number of nodes visited and the higher the number of correctly solved tasks. The number of nodes was also shown to affect the number of correctly solved tasks. This may be related to interface choice: Whereas the participants with a higher ability to concentrate used link following and keyword specification in a balanced way, the participants with a lower ability to focus most often tried to reach their goal by typing

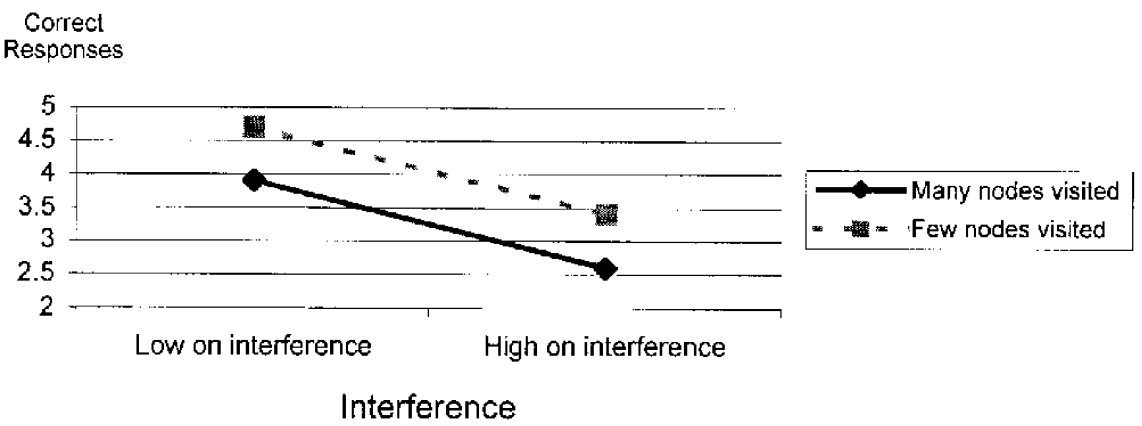

Figure 6. Correct responses by interference group and nodes visited (Experiment 1). 


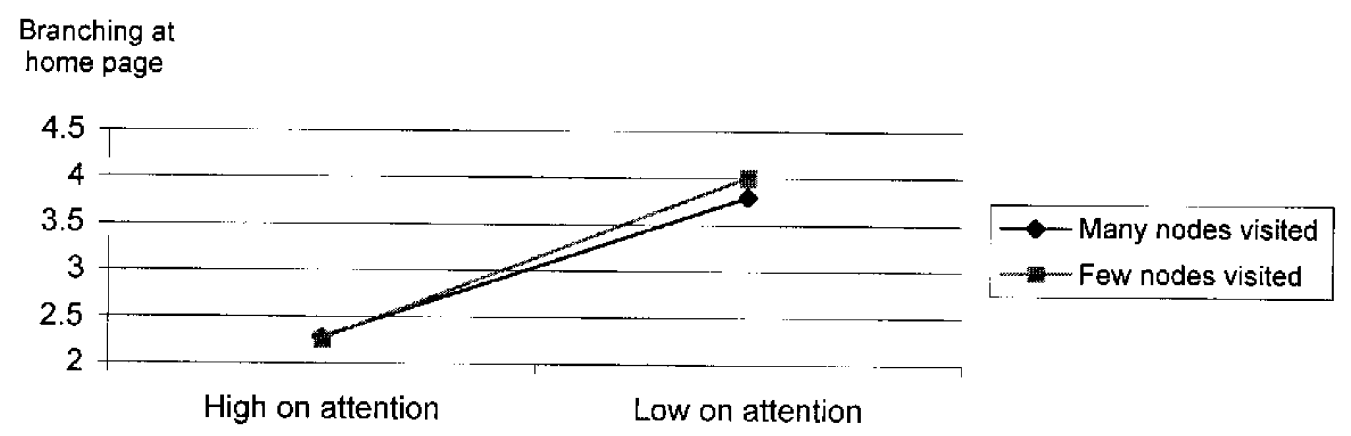

Attention

Figure 7. Branching at home page by attention group and nodes visited (Experiment 2).

a search term directly at the start page. Keyword specification proved to be less efficient than the careful choice of a good overview page.

In summary, each of the measures defined on theoretical grounds was shown to have a distinctive realization in the graphs, and this structural preordering helped us evaluate the different solution paths qualitatively.

Further research will extend the STRATDYN approach in two respects. First, STRATDYN operates both with quantitative measures and patterns and with visualizations (see above and Berendt, 2000). In the future, more sophisticated quantitative measures of navigation behavior will be developed. Structurally, these will rely on the modeling of sequences in terms of grammars (Olson et al., 1994; see also Fu, 2001). Equally important, ways of taking domain knowledge into account will be explored. Domain knowledge about the site and the task has been utilized in the part of STRATDYN presented in Berendt. It allowed us to find statistically significant differences between the navigation behavior of different groups of users and to visualize these differences. We expect that the integration of domain knowledge will allow us to derive more detailed and powerful measures of navigation behavior. Second, we are also exploring ways of combining the analysis of individual users' behavior with the methodology used to analyze data gathered in the normal operation of the server - that is, field data coming from a very large group of users (Berendt, 2000).

In the present study, we have concentrated on the analysis of Web page request sequences and have ignored the time intervals between them. Although the analysis of times needed for the appreciation of different hypertext pages is desirable, Web server data are not a good source of such time data. This is because Internet traffic influences the time between the sending of the page by the server (this is recorded) and the delivery to the user's computer. Client-side data collection is needed for more reliable data. This option will be explored in the future.

Client-side or proxy server data collection will also enable us to observe navigation behavior across multiple servers. The question is whether and to what extent the different servers' information architectures influence a given individual's search technique. We expect that individual differences in cognitive/learning styles will have an effect here too. For example, it is possible that fielddependent individuals will adapt their search techniques much more to the server's information architecture than will field-independent individuals.

We have shown that individual differences in the ability to focus one's attention on relevant cues and to judiciously discard potentially distracting stimuli may account for differences in performance. Future research should examine to what extent differences in attentional factors are related to other individual difference variables that have been shown to affect navigational behavior, such as field dependence or other cognitive styles (Riding \& Sadler-Smith, 1992), spatial ability (Dahlbäck, Höök, \& Sjölinder, 1996; Höök, Sjölinder, \& Dahlbäck, 1996), or learning style and strategies (Hammond, 1993; Schellhas \& Brenstein, 1998). Certainly, feelings of disorientation may also result from poor structure or lack of content expertise. The interaction of attentional factors with interface structure or prior knowledge should be examined. As more is known about specific navigational strategies of individuals with different attentional abilities, recommendations should be formulated to aid different groups of learners when they are interacting with the Web. Future research should especially examine the effectiveness of interventions aimed at reducing the persistent problem of navigational disorientation, keeping in mind individual abilities and style preferences of a diverse user population.

\section{REFERENCES}

Barab, S. A., Bowdish, B. E., \& Lawless, K. A. (1997). Hypermedia navigation: Profiles of hypermedia users. Educational Technology Research \& Development, 45, 23-42.

BäUMLER, G. (1985). Farb-Wort-Interferenztest (FWIT) nach J. R. Stroop. Handanweisung [Color-word interference test following J. R. Stroop: Manual]. Göttingen: Hogrefe.

BerEndT, B. (2000). Web usage mining, site semantics, and the support of navigation. In R. Kohavi, M. Spiliopoulou, J. Srivastava, \& 
B. Masand (Eds.), Working notes of the workshop "Web Mining for E-Commerce-Challenges \& Opportunities": 6th ACM SIGKDD International Conference on Knowledge Discovery and Data Mining (pp. 83-93). New York: ACM Press.

Berendt, B., RAuH, R., \& Barkowsky, T. (1998). Spatial thinking with geographic maps: An empirical study. In H. Czap, H.-P. Ohly, \& S. Pribbenow (Eds.), Herausforderungen an die Wissensorganisation: Visualisierung, multimediale Dokumente, Internetstrukturen (Proceedings ISKO'97) (pp. 63-74). Würzburg: ERGON-Verlag.

Berendt, B., \& Spiliopoulou, M. (2000). Analysing navigation behavior in Web sites integrating multiple information systems. $V L D B$ Journal, 9, 56-75.

Bertin, J. (1983). Semiology of graphics. Madison: University of Wisconsin Press.

BRENSTEIN, E. (1996). Untersuchungsmöglichkeiten von Lernverhalten in hypermedialen Lernumgebungen [Investigating learning behavior in hypermedia learning environments]. In Interdisziplinäres Zentrum für Lern- und Lehrforschung (Ed.), LLF-Berichte No. 16 (pp. 45-55). Potsdam: University of Potsdam.

Brenstein, E. \& BeRENDT, B. (2001). Individual differences in attention and Web navigation. Unpublished manuscript, Institute of Pedagogy and Informatics, Humboldt University, Berlin.

BRICKENKAMP, R. (1994). Aufmerksamkeit, Konzentration und Arbeitsverhalten. Aufmerksamkeits-Belastungs-Test (d2) [Attention, concentration, and work behavior: Attention-test (d2)]. Göttingen: Hogrefe.

CANTer, D., Rivers, R., \& Storrs, G. (1985). Characterizing user navigation through complex data structures. Behavior \& Information Technology, 4, 93-102.

Card, S. K., Mackinlay, J. D., \& Shneiderman, B. (1999). Information visualization. In S. K. Card, J. D. Mackinlay, \& B. Shneiderman (Eds.), Readings in information visualization: Using vision to think (pp. 1-34). San Francisco: Morgan Kaufmann.

CHEN, C. (1999). Information visualisation and virtual environments. London: Springer-Verlag.

Chen, C., \& RADA, R. (1996). Interacting with hypertext: A metaanalysis of experimental studies. Human-Computer Interaction, 11, 125-156.

Conklin, J. (1987). Hypertext: An introduction and survey. Computer Magazine, 20, 17-41.

DAhlbäck, N., HööK, K., \& SJölinder, M. (1996). Spatial cognition in the mind and in the world: The case of hypermedia navigation. In G. W. Cottrell (Ed.), Proceedings of the Eighteenth Annual Meeting of the Cognitive Science Society (pp. 195-200). Mahwah, NJ: Erlbaum.

Dillon, A., McKnight, C., \& Richardson, J. (1993). Space-the final chapter or: Why physical representations are not semantic intentions. In C. McKnight, A. Dillon, \& J. Richardson (Eds.), Hypertext: A psychological perspective (pp. 169-191). Chichester, U.K.: Ellis Horwood.

Dufresne, A., \& Turcotte, S. (1997). Cognitive style and its implications for navigation strategies. In B. D. Boulay \& R. Mizoguchi (Eds.), Artificial intelligence in education: Knowledge and media in learning systems (pp. 287-293). Amsterdam: IOS Press.

Edwards, D., \& Hardman, L. (1989). "Lost in hyperspace": Cognitive mapping and navigation in a hypertext environment. In R. McAleese (Ed.), Hypertext: Theory into practice (pp. 105-125). Oxford: Intellect.

Ellis, R. D., Jankowski, T. B., Jasper, J. E., \& Tharuvai, B. S. (1998). Listener: A tool for client-side investigation of hypermedia navigation behavior. Behavior Research Methods, Instruments, \& Computers, 30, 573-582.

FU, W.-T. (2001). ACT-PRO action protocol analyzer: A tool for analyzing discrete action protocols. Behavior Research Methods, Instruments, \& Computers, 33, 149-158.

GinSBERG, M. L. (1993). Essentials of artificial intelligence. San Francisco: Morgan Kaufman.

Hall, R. H., Balestra, J., \& Davis, M. (2000, April). A navigational analysis of linear and non-linear hypermedia interfaces. Paper presented at the Annual Meeting of the American Educational Research Association, New Orleans.

Hammond, N. (1993). Learning with hypertext: Problems, principles, and prospects. In C. McKnight, A. Dillon, \& J. Richardson (Eds.), Hypertext: A psychological perspective (pp. 51-70). London: Ellis Horwood:

Hasebrook, J., \& Fezzardi, G. (1996). Learning with hypermedia. What users do and how to observe it automatically. In P. Carlson \& F. Makedon (Eds.), Proceedings of ED-MEDIA 96 \& ED-TELEKOM 96-World Conference on Educational Multimedia and Hypermedia and World Conference on EducationalTelecommunications (pp. 10281029). Charlottesville, VA: Association for the Advancement of Computing in Education.

Hemmje, M., KunKel, C., \& Willett, A. (1994). Lyberworld: A visualization user interface supporting fulltext retrieval. In W. B. Croft \& C. J. van Rijsbergen (Eds.), Proceedings of the Seventeenth Annual InternationalACM-SIGIR Conference on Research and Development in Information Retrieval (pp. 249-259). London: Springer-Verlag.

HööK, K., SJölINDER, M., \& DAHLBäCK, N. (1996). Individual differences and navigation in hypermedia. In T. R. G. Green, J. J. Canas, \& C. P. Warren (Eds.), Proceedings of the Eighth European Conference on Cognitive Ergonomics (ECCE-8) (pp. 195-200). Grenada, Spain: University of Granada Press.

KIM, K.-S. (2000). Effects of cognitive style on Web search and navigation. In J. Bourdeau \& R. Heller (Eds.), ED-MEDIA 2000: Proceedings of the World Conference on Educational Multimedia, Hypermedia and Telecommunications (pp. 496-501). Charlottesville, VA: Association for the Advancement of Computing in Education.

MACGregor, S. K. (1999). Hypermedia navigation profiles: Cognitive characteristics and information processing strategies. Journal of Educational Computing Research, 20, 189-206.

Mackinlay, J. (1986). Automating the design of graphical presentations of relational information. ACM Transactions on Graphics, 5, 110-141.

Mukherjea, S., \& Hara, Y. (1997). Focus+context views of WorldWide Web nodes. In M. Bernstein, L. Carr, \& K. Dsterbye (Eds.), Hypertext' 97: The Eighth ACM Conference on Hypertext (pp. 187196). New York: ACM Press.

MUnZnER, T. (1998). Exploring large graphs in hyperbolic space. IEEE Computer Graphics \& Its Applications, 18, 18-23.

Newell, A., \& Simon, H. A. (1972). Human problem solving. Englewood Cliffs, NJ: Prentice-Hall.

Newfield, D., Sethi, B. S., \& Ryall, K. (1998). Scratchpad: Mechanisms for better navigation in directed Web searching. In Proceedings of the Eighth Annual ACM Symposium on User Interface Software and Technology (pp. 1-8). New York: ACM Press.

NiegemanN, H. M. (2000, April). Analyzing processes of self-regulated hypermedia-supported learning: On the development of a log-file analysis procedure. Paper presented at the Annual Meeting of the American Educational Research Association, New Orleans.

Nielsen, J. (2000). Designing Web usability: The practice of simplicity. Indianapolis, IN: New Riders Publishing.

Olson, G. M., Herbsleb, J. D., \& Rueter, H. (1994). Characterizing the sequential structure of interactive behaviors through statistical and grammatical techniques. Human-Computer Interaction, 9, 427-472.

Pirolli, P., \& CARD, S. K. (1995). Information foraging in information access environments. In Proceedings of the Conference on Human Factors in Computing (CHI 95) (pp. 51-58). New York: ACM Press. Riding, R, \& SADLER-Smith, E. (1992). Type of instructional material, cognitive style and learning performance. Educational Studies, 18, 323-340.

Schellhas, B., \& BREnStein, E. (1998). Learning strategies in hypermedia learning environments. In T. Ottmann \& I. Tomek (Eds.), EDMEDIA and ED-TELEKOM 98: Proceedings of the 10th World Conference on Educational Multimedia and Hypermedia and World Conference on Educational Telecommunications (pp. 1922-1923). Charlottesville, VA: Association for the Advancement of Computing in Education.

ShNEIDERMAN, B. (1998). Designing the user interface. Reading, MA: Addison-Wesley.

Siegel, A., \& White, S. (1975). The development of spatial representations of large-scale environments. In H. Reese (Ed.), Advances in child development and behavior (pp. 9-55). New York: Academic Press. 
Sorrows, M. E., \& Hirtle, S. C. (1999). The nature of landmarks for real and electronic spaces. In C. Freksa \& D. Mark (Eds.), Spatial information theory: Proceedings International Conference COSIT '99 (pp. 37-50). Berlin: Springer-Verlag.

SpILIOPOUlou, M. (1999). The laborious way from data mining to Web mining. International Journal of Computer Systems, Science \& Engineering, 14, 113-126.

Srivastava, J., Cooley, R, DeshPande, M., \& Tan, P.-N. (2000). Web usage mining: Discovery and application of usage patterns from Web data. SIGKDD Explorations, 1, 12-23.

TAuscher, L., \& Greenberg,S. (1997). How people revisit Web pages: Empirical findings and implications for the design of history systems. International Journal of Human Computer Studies, 47, 97-138.

Tufte, E. R. (1997). Visual explanations. Cheshire, CT: Graphics Press. TukEY, J. W. (1977). Exploratory data analysis. Reading, MA: AddisonWesley.

Unz, D. C., \& Hesse, F. W. (1999). The use of hypertext for learning. Journal of Educational Computing Research, 20, 279-295.

Wandke, H., \& Hurtienne, J. (1998). Praxiskonzepte und Erfahrungsberichte: Zum Navigationsverhalten von Anfängern im World-Wide Web [Concepts for practice and experience reports: On novices' navigation behavior on the World-Wide Web]. Zeitschrift für Arbeits- und Organisationspsychologie, 43, 46-54.

Witten, I. H., \& Frank, E. (2000). Data mining: Practical machine learning tools and techniques with Java implementations. San Diego: Academic Press.

WRIGHT, P. (1991). Cognitive overheads and prostheses: Some issues in evaluating hypertexts. In Proceedings of the 3rd ACM Conference on Hypertext (pp. 1-12). New York: ACM Press.

\section{NOTES}

1. The term search is used in three ways. The first meaning, and the one used here, is from the problem-solving literature (e.g., Newell \& Simon, 1972) and refers to "the search for a solution to a problem, no matter how it is done."

The second meaning derives from the term "search in databases" and is therefore often used synonymously with the typing in of keywords, as opposed to the following of a link (cf. the quotations from Nielsen, 2000 , below). We will refer to this dimension as interface choice and distinguish between keyword specification and link following.

The third goes back to Canter et al. (1985, p. 100), who distinguish navigation strategies-for example, searching by "users motivated to find a particular target" and browsing, "where users are happy to go wherever the data takes them until their interest is caught." This distinction is also known as directed search versus undirected search (e.g.,
Newfield, Sethi, \& Ryall, 1998). Our approach encompasses all these strategies, and in the empirical experiments described below, we motivated participants to find a particular target by setting tasks. Therefore, this distinction of navigation strategies would not contribute to the discussion in the present paper.

2. The notion of Web search used here encompasses all intentional activities with an at least minimally specified information need. "Surfing" the Web for potentially interesting material on arbitrary subjects is excluded from the definition.

3. Nielsen (2000, p. 224) found that "slightly more than half of all users are search-dominant, about a fifth of the users are link-dominant, and the rest exhibit mixed behavior.' Search-dominant users prefer the use of the search button and keyword specification, whereas linkdominant users prefer to follow the links around a site.

4. For example, in Spiliopoulou (1999), the basic knowledge representation form is a sequence, but a node specif ied in the search for patterns can be constrained by the count of prior visits to this node in the sequence. This is equivalent to our labeling of edges in the navigation graph.

5. The exact formal notation and the pattern-matching algorithm have been omitted to increase legibility.

6. For two impressive real-world examples, the discovery of the origins of cholera as well as the Challenger spaceship disaster, see Tufte, 1997.

7. Canter et al. (1985) use the ratio of number of nodes visited to total number of nodes available in the system. In a Web-based experiment, the number of nodes available is the total number of pages in one server or in the whole Web. In either case, this number is so large that using it for normalization would obscure any differences between individuals.

8. Barab, Bowdish, and Lawless (1997) measure the maximum depth of exploration. However, this may be misleading because of possible outliers. We were more interested in the average behavior over tasks.

9. This is an adaptation of the formula proposed by Mukherjea and Hara (1997) to navigation graphs for discovering landmarks in a site. Their formula includes three components: connectivity, frequency of access, and depth in the file system. Frequency of access is equivalent to connectivity in navigation graphs. Depth in the file system is important for evaluating a node in the context of a site, because users often type in URLs, favoring short URLs. It is less relevant in evaluating a node in the context of a navigation graph, since URLs are most often reached via hyperlinks.

The measure is also an extension of Canter et al.'s (1985) ratio of the number of distinct nodes visited to the total number of nodes visited.

(Manuscript received November 29, 2000; revision accepted for publication March 20, 2001.) 